\title{
Estado del arte. Un mundo tecnológico cambiante
}

\author{
Dr. Ricardo Díaz-Guillén \\ Cirujano Plástico, Panamá. \\ Director del Capítulo de Procedimientos Estéticos no Quirúrgicos de la FILACP 2014-2016
}

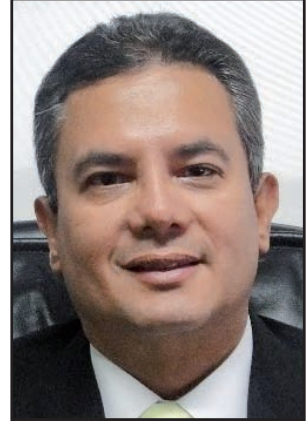

Díaz-Guillén, $\mathbf{R}$.
Cuando el Dr. Julio Kirschbaum, Presidente de la FILACP, me encomendó la tarea de escribir sobre este tópico, no pensé en la dificultad que tendría para encontrar literatura científica al respecto. Vaya sorpresa, porque ni en los libros, ni en las revistas científicas de las especialidades que tienen que ver con el tema, encontré artículos que me sirvieran. Creo que esto se debe principalmente a que las tecnologías punteras van cambiando rápidamente, a que hay mucha competencia entre los fabricantes, y a que solo hay experiencias clínicas individuales. Por tal motivo, decidí dirigir mi investigación en otra vía, y poder así brindarles un pantallazo de algunas de las tecnologías que existen hoy en día en el mercado y que si bien mañana quizás ya sean obsoletas, hoy por hoy tal vez puedan ser de utilidad en nuestra práctica clínica como una herramienta adicional para ofrecer a nuestros pacientes.

A medida que el mercado de los equipos estéticos sigue evolucionando, es un desafío conocer hacia dónde se dirigen estos cambios en nuestra especialidad. En los últimos años varias de estas tecnologías han ido ganando gran popularidad. Si bien el énfasis se centra en ciertos procedimientos, las mejoras graduales están abarcando cada vez un espectro mucho más amplio; por lo tanto es muy importante que los fabricantes de estos equipos y los usuarios, por igual, tengamos en cuenta el creciente potencial de los mismos.

Los nuevos dispositivos han proporcionado sin duda sus mayores avances en el campo de la estética, sobre todo en el estiramiento cutáneo y en la reducción no invasiva de la grasa corporal. El estiramiento cutáneo facial, principalmente, ha despertado mucho interés por parte de las compañías que fabrican estos equipos, ya que su uso ha superado las expectativas en los resultados clínicos en cuanto a previsibilidad y satisfacción de los pacientes, lo que ha motivado su mayor utilización. Es el caso del equipo Thermage $^{\circledR}$ (Solta Medical Inc.; San Francisco, California, EE.UU) basado en el uso de radiofrecuencia, o el Ultherapy ${ }^{\circledR}$ (Ulthera Inc.; Mesa Arizona, EE.UU) que parece, en general, proporcionar mejores resultados con menos dolor.

Ultherapy® usa la energía del ultrasonido generando calor y contrayendo el tejido dérmico sin alterar la superficie cutánea; es la única tecnología aprobada por la Food and Drugs Administration de los EE.UU. (FDA) para el estiramiento cutáneo a nivel de cejas, región mentoniana y cuello.

Su mecanismo de acción, a base de la emisión de calor multifocal, calienta los tejidos a una temperatura óptima de entre 65 y $70{ }^{\circ} \mathrm{C}$ durante un lapso determinado de tiempo, lo que induce una necrosis coagulativa de gran precisión gracias al sistema visual de ultrasonido, que permite localizar el calor desde $1 \mathrm{~mm}$ (epidermis) a 4,5 mm (sistema muscular superficial de la cara y cuello). Así consigue que miles de diminutos y precisos puntos de coagulación, a múltiples profundidades, creen una primera etapa de desnaturalización del colágeno dando inicio a la cascada de la neocolagénesis. Esta respuesta implica la síntesis de nuevo colágeno, lo que permite a la piel tener mejores propiedades viscoelásticas y resistir mejor las tensiones mecánicas, lo que con el tiempo conlleva a su vez una mayor firmeza de los tejidos.

Otro de los equipos que ha estado en el mercado por poco tiempo y que ha dado muy buena impresión para el estiramiento facial es el PrecisionTX ${ }^{\circledR}$ (Cynosure Inc.; Westford, Massachusetts, EE.UU.), también aprobado por la FDA, que se utiliza para el tratamiento del contorno del tercio inferior de la cara y del cuello, aunque sus resultados se ven a más largo plazo.El Thermi $\mathrm{RF}^{\circledR}$ (Thermi Inc; Irving, Texas, EE.UU.) es otro de los aparatos aprobados por la FDA que recientemente ha ganado atención, y que emplea una cánula que se coloca bajo la piel para calentarla con una cámara térmica.

Si bien las mejoras graduales de los dispositivos para el tratamiento de la piel han logrado captar la atención, más populares han llegado a ser aquellos equipos destinados a lograr la reducción no invasiva de la grasa corporal. Varias tecnologías de este tipo están ya en el mercado, incluyendo la que utiliza la criolipólisis, como CoolSculpting ${ }^{\circledR}$ (Zeltiq Aesthetic Inc.; Pleasanton, California, EE.UU.); el ultrasonido, como LipoSonix ${ }^{\circledR}$ (Solta Medical Inc.; San Francisco, California, EE.UU); la radiofrecuencia, como Exilis $^{\circledR}$, y más recientemente el Vanquish ${ }^{\circledR}$ (ambos de BTL Industries, Ltd.; Cleveland, Ohio, EE.UU), que calientan selectivamente el tejido adiposo hasta el punto de la apoptosis, afectando menos al tejido circundante.

Con una variedad más amplia de tratamientos, estas tecnologías de remoción de la grasa de forma no invasiva son una nueva herramienta que se puede ofrecer como alternativa para aquellos pacientes que, si bien podrían beneficiarse más de una liposucción, se resisten a someterse a cualquier tipo de práctica invasiva, o para aquellos pacientes que ameritan poca remoción de la grasa focalizada.

CoolSculpting ${ }^{\circledR}$ es hasta la fecha el único procedimiento aprobado por la FDA para el tratamiento no quirúrgico de la grasa corporal. Utiliza el enfriamiento controlado para eliminar la grasa localizada a través del congelamiento y muerte de los adipocitos; sobre todo en aquellos depósitos de grasa que son rebeldes al tratamiento mediante dieta y/o ejercicio, con resultados que parecen ser notables y duraderos.

Estos procedimientos de tratamiento del contorno corporal han demostrado ser financieramente rentables, pero científicamente aún es mucho lo que queda por demostrar sobre su eficacia.

Las tecnologías basadas en el uso de láseres fraccionales han tenido también un gran auge en los últimos tiempos, como lo demuestra el gran número de dispositivos existentes en la actualidad en el mercado. Uno de ellos es el Halo ${ }^{\circledR}$ (Sciton Inc.; Palo Alto, California, EE.UU.) que ofrece longitudes de onda ablativas y no ablativas en la misma pieza de mano. Permite que los respectivos microhaces ablativos y no ablativos puedan ser desplegados adyacentemente o uno encima del otro, dependiendo de las preferencias del usuario.

Por su parte, los equipos de radiofrecuencia siguen siendo muy populares ya que tienden a ser más baratos, y es muy probable que sigan creciendo en el mercado dado que su fabricación es muy rentable y proporcionan resultados razonablemente eficaces en su conjunto. De este tipo son eMatrix ${ }^{\circledR}$ con tecnología de radiofrecuencia bipolar fraccionada (Syneron Medical Ltd.; Yokneam Illit, Israel) y Pixel $\mathrm{RF}^{\circledR}$ de tecnología de microplasma de radiofrecuencia fraccionada, (Alma Lasers Medical; Israel). Pero como 
sucede con cualquiera tecnología, no hay un tipo especifico de estos equipos que trate toda la gama de condiciones existentes y probablemente, veremos florecer aún más tecnologías no ablativas.

La eliminación de los tatuajes corporales es otra de las áreas específicas de tratamientos en la que se utilizan métodos no invasivos; sin embargo, sigue siendo un área de difícil incursión. Recientemente, la tecnología de picosegundos del láser PicoSure ${ }^{\circledR}$ de Cynosure Inc ha logrado reducir el tiempo de exposición del haz de luz, ya que produce pulsos ultracortos en un tiempo de una billonésima de segundo; esta duración ultracorta de PicoSure ${ }^{\circledR}$ crea una acción fototérmica generando un impacto fotomecánico (PressureWave $\left.^{\circledR}\right)$, y originando así una mayor acción en el área a tratar que hace que sean necesarias menos sesiones de tratamiento en comparación con los láseres tradicionales, cuya tecnología de nanosegundos está basada principalmente en la acción fototérmica entregando calor al pigmento y al tejido circundante. Esto ha cambiado el panorama mediante la posibilidad de reducir el número de tratamientos necesarios para la eliminación de algunos tatuajes.

Sin embargo, para que estos dispositivos puedan ser más exitosos y ampliamente incorporados a la práctica diaria, tienen que hacer algo más que la simple eliminación de los tatuajes considerando lo costosos que son estos equipos. Afortunadamente, la tecnología de picosegundos tiene una utilidad más amplia de lo que se pensaba ya que parece que puede utilizarse también, potencialmente, para el rejuvenecimiento facial. En una aplicación, el haz del láser de picosegundos es similar al haz de un láser fraccionado, pero éste lo hace en muchos impulsos individuales. El resultado es un rejuvenecimiento más eficaz sin mucho tiempo de inactividad para el paciente. Por todo ello, podemos considerar que si bien la tecnología ideal para la eliminación de tatuajes aún no se ha descubierto, la tecnología de picosegundo supone un importante paso en la dirección correcta.

En cuanto a otros avances, un área que continuamente está siendo investigada en cuanto al uso del tratamiento con láser es el acné. En concreto, algunas empresas están empezando a ver cómo ciertos dispositivos que se habían desarrollado inicialmente para tratamientos cosméticos, también pueden tener utilidad para el tratamiento del acné y de las cicatrices. La dificultad está en la obtención del reembolso por parte de los seguros médicos, que hasta ahora ha sido un obstáculo muy difícil de superar, si bien algunas compañías de seguros y proveedores están involucrándose en llevar a cabo esfuerzos por integrar los nuevos códigos CPT de estos tratamientos, y así logar que el coste sea reembolsado a los pacientes.

Otro ejemplo de estas dificultades es la aprobación de la herramienta MiraDry (MiraWave ${ }^{\circledR}$, Miramar Labs, Inc; Santa Barbara, California, EE.UU.), uno de los dispositivos más eficaces para el tratamiento de la hiperhidrosis, que utiliza energía a través de microondas para destruir las glándulas sudoríparas (zona de calor); una vez destruidas no vuelven a regenerarse, lo que hace que el tratamiento sea permanente. Al mismo tiempo el dispositivo aplica enfriamiento superficial (zona fría) que protege la piel y es calmante.

Un elemento clave que debería ayudar en la integración de estos equipos en nuestra práctica clínica es su fiabilidad. A menudo, los dispositivos más exitosos son aquellos que son fáciles de manejar y las plataformas a las que se les pueden incorporar herramientas que mejoren los resultados, como por ejemplo, el que puedan proporcionar información en tiempo real y aumentar así la confianza en la selección de parámetros. Todos estos tipos de avances harán que progresivamente, su uso sea más convencional. Los dispositivos que son seguros para operar y fáciles de navegar continuarán atrayendo la mayor atención y reducirán la brecha de su uso en nuestra especialidad.

Con las innovaciones continuas en el ámbito de los procedimientos basados en este tipo de dispositivos, los cirujanos plásticos tenemos muchas opciones que podemos incorporar a nuestra práctica médica para agregar un valor más a nuestras consultas. Pero al mismo tiempo que cada uno de estos dispositivos ofrece a los pacientes ventajas únicas, también tienen limitaciones específicas que tanto los médicos como los pacientes debemos tener en cuenta antes de invertir en ellos. Estas limitaciones van desde los honorarios médicos hasta la imprevisibilidad de los resultados. Teniendo en cuenta que esta área de tratamiento es aún muy joven, debemos esperar un crecimiento continuo y dar más seguimiento a la viabilidad de muchos de estos dispositivos desde un punto de vista científico. 